\title{
FUNGO MICORRÍZICO, FÓSFORO E NITROGÊNIO NO CRESCIMENTO INICIAL DA TREMA E DO FEDEGOSO(1)
}

\author{
M. E. PARON ${ }^{(2)}$, J . O. SIQUEIRA ${ }^{(3)} \&$ N. CURI ${ }^{(3)}$
}

\begin{abstract}
RESUMO
De março de 1993 a junho de 1994, em vasos com amostra de um latossolo vermelho-escuro (LE) argiloso fase cerrado, estudou-se a resposta da trema (Trema micrantha (L)Blum.) e do fedegoso (Senna macranthera Rich.) a fósforo (P) nitrogênio (N) eà inoculação com o fungo mi corrízico arbuscular Glomus etunicatum (Ge) Becker \& Gerdemann. 0 trabalho foi desenvolvido em casa de vegetação do Departamento de Ciência do Solo da Universidade Federal de Lavras (MG), por meio de dois experimentos. A inoculação com Ge resultou em efeitos positivos para o crescimento das espécies, sendo esse efeito menos evidente no fedegoso que se beneficiou mais dos fungos indígenas. $\mathrm{E}$ plantas inoculadas, a dose de $\mathrm{P}$ necessária para atingir $80 \%$ do crescimento máximo (CM) foi de $100 \mathrm{mg} \mathrm{kg}^{-1}$ de P no solo, para a trema, e de $80 \mathrm{mg} \mathrm{kg}^{-1}$ de P no solo, para o fedegoso, enquanto as plantas colonizadas pelos fungos indígenas requereram, respectivamente, 3,2 e 1,5 vezes mais P para atingir tal crescimento. Ambas as espécies apresentaram crescimento reduzido na ausência de $P$, porém tiveram grande crescimento quando receberam superfosfato. Por outro lado, a adição de $\mathbf{N}$ mineral não promoveu o crescimento das mudas. A adição de $P$ solúvel $e$ a introdução de $G$. etunicatum são importantes fatores para o crescimento inicial das espécies estudadas, em solo de baixa ferti lidade natural.
\end{abstract}

Termos de indexação: essências nativas, florestamento, micorriza arbuscular, fungos de solo, Glomus etunicatum.

\section{SUMMARY: MYCORRHIZAE, PHOSPHORUS AND NITROGEN SUPPLY ON INITIAL GROWTH OF "TREMA" AND "FEDEGOSO"}

Theresponseof "trema" (Trema micrantha (L) Blum.) and "fedegoso" (Senna macranthera Rich.), both native woody species, to phosphorus (P), nitrogen (N) and inoculation with arbuscular mycorrhizal fungus (AMF) was studied in pots using a low-fertility clayey Dark Red-Latosol (Oxisol) Sample The study, composed of two experiments, was carried out under

\footnotetext{
(1) Trabal ho Parcialmente financiado pela CEMIG e FAPEMIG. Recebido para publicação em janeiro e aprovado em outubro de 1997.

(2) Engenheiro-Agrônomo, Centro de Pesquisa do Trópico Semi-Árido - EMBRAPA, Caixa Postal 23, CEP 56300-000 Petrolina (PE). Bolsista do CNPq.

(3) Engenheiro-Agrônomo, Professor Titular do Departamento de Ciência do Solo, UFLA, Caixa Postal 37, CEP $37200-000$ Lavras (MG). Bolsista do CNPq.
} 
greenhouseconditions at theSoil ScienceDepartment of UniversidadeF ederal deLavras, state of Minas Gerais, Brazil, from march 1993 to april 1994. Both species grew poorly in theabsence of added $P$ and exhibited great responseto this fertilizer. Inoculation with Glomus etunicatum Becker \& Gerd. (Ge) resulted in benefi cial plant growth effects in both species. I nocul ated plants reached $80 \%$ of themaximum growth when $100 \mathrm{mg} \mathrm{kg}^{-1}$ of $\mathrm{P}$ in thesoil was added to trema and of $80 \mathrm{mg} \mathrm{kg}^{-1}$ of $\mathrm{P}$ in the soil to fedegoso. Trema and fedegoso plants non-inoculated with $\mathrm{Ge}$ but col onized by indigenous fungi, required 3.2 and 1.5 times, respectively, moreP to reach $80 \%$ of maxi mum growth. Addition of mineral $\mathrm{N}$ alonedid not promoteplant growth of any species. Theaddition of solublephosphateand the presence of AMF propagules are of great importance for initial growth of the studied species in low-fertility soils.

Index terms: native woody species, forestation, arbuscular mycorrhiza, soil fungi, Glomus etunicatum.

\section{INTRODUÇÃO}

As deficiências nutricionais constituem as principais limitações ao crescimento de espécies arbóreas nativas em áreas de sol o de baixa fertilidade natural. Espécies nativas do sudeste brasileiro respondem, positivamente, à adubação com macronutrientes, e a omissão de nitrogênio e fósforo da adubação completa restringe o crescimento inicial nos solos predominantes na região Campos das Vertentes, sobretudo os da área sob influência da usina hidrelétrica I tutinga/Camargos (Siqueira et al., 1995; Renó et al., 1997). Diversos estudos relacionam os requerimentos nutricionais de espécies arbóreas à presença de simbioses mutualistas, como as mi corrizas arbusculares e a nodulação pelo rizóbio, capazes de al iviar os estresses nutricionais impostos por sol os de baixa fertilidade (Franco, 1984; Col onna et al., 1991; Pereira et al., 1996a). As micorrizas, além de aumentar a absorção de fósforo, nitrogênio e outros nutrientes do solo, favorecem a fixação biológica de $\mathrm{N}_{2}$ nas leguminosas nodulíferas (Siqueira, 1990). Entretanto, pouco se conhece sobre os efeitos da simbiose micorrízica em espécies arbóreas nativas, como a trema (Trema micrantha (L.) Blum.) e o fedegoso (Senna macranthera Rich.).

A trema éuma espécie arbórea pioneira da família Ulmaceae, de ocorrência natural nas formações florestais da Bahia ao Rio Grande do Sul, utilizada em ripados e, como forrageira (Lorenzi, 1992), em plantios mistos, visando à recuperação de ecossistemas degradados (Carvalho, 1994). A pesar da sua ocorrência generalizada e do interesse sobre essa espécie, pouco se conhece sobre suas exigências nutricionais (Gonçalves et al., 1992).

O fedegoso é uma leguminosa não-nodulífera de hábito pioneiro, que ocorre em formações florestais do Ceará até São Paulo (Lorenzi, 1992). É empregado apenas em caixotaria, brinquedos e como lenha, apresentando grande potencial para uso no reflorestamento de áreas degradadas. Tal como verificado para a trema, as exigências nutricionais dessa espécie são pouco conhecidas. Sabe-se, entretanto, que Lima (1995) obteve el evada resposta dessa leguminosa à adubação mineral com macronutrientes em um latossol o de baixa fertilidade natural de Minas Gerais, bem como verificou que a omissão de $\mathrm{N}$ ou $\mathrm{P}$ reduziu, expressivamente, o crescimento das plantas até 24 meses após o plantio.

Tanto a trema quanto o fedegoso formam associações micorrízicas arbusculares (St. J ohn, 1980; Carneiro et al., 1996), sendo essencial a précolonização de mudas, visando a plantios em áreas pobres em nutrientes, para garantir seu estabelecimento e desenvolvimento no campo. O objetivo do presente estudo foi avaliar em casa de vegetação a resposta dessas espécies à adubação com fósforo, nitrogênio mineral eà inoculação com o fungo micorrízico arbuscular Glomus etuni catum Becker \& Gerdemann em amostras de solo de baixa fertilidade natural.

\section{MATERIAL E MÉTODOS}

O estudo, composto por dois experimentos, foi desenvol vido em casa de vegetação do Departamento de Ciência do Solo (DCS) da Universidade Federal de Lavras (MG) (UFLA), utilizando-se amostras da camada arável de um latossolo vermel ho-escuro argil oso fase cerrado, col etado próximo da represa de Itutinga/Camargos (MG). A análisequímica apresentou os seguintes resultados: carbono orgânico, extraído com $\mathrm{Na}_{2} \mathrm{Cr}_{2} \mathrm{O}_{7} 2$ mol L-1 $+\mathrm{H}_{2} \mathrm{SO}_{4} 5$ mol L-1 edeterminado por colorimetria $=27 \mathrm{~g} \mathrm{~kg}^{-1} ; \mathrm{pH}$ em água $(1: 2,5)=$ 5,2; $\mathrm{Ca}=2 \mathrm{mmol}_{\mathrm{C}} \mathrm{dm}^{-3}, \mathrm{Mg}^{\prime}=1 \mathrm{mmo}_{\mathrm{C}} \mathrm{dm}^{-3} \mathrm{eAl}=$ $7 \mathrm{mmol}_{\mathrm{c}} \mathrm{dm}^{-3}$, extraídos com KCl $1 \mathrm{~mol} \mathrm{~L}^{-1} \mathrm{e}$ determinados por titulometria; $\mathrm{P}=2 \mathrm{mg} \mathrm{kg}^{-1}, \mathrm{~K}=$ $42 \mathrm{mg} \mathrm{kg}^{-1}$, extraídos pelo Mehlich-1, sendo os teores de $\mathrm{P}$ determinados por colorimetria, e os de K, por fotometria de chama. Obteve-se teor de massa orgânica (43 g kg-1) de maneira indireta, com base no teor de carbono orgânico $(1,6 \mathrm{x})$. O material de solo, col etado na camada de $0-20 \mathrm{~cm}$, foi peneirado (malha de $2 \mathrm{~mm}$ ) edeixado secar ao ar. A densidade de esporos de fungos micorrízicos arbusculares (MAs) no sol o foi determinada, conforme Gerdemann \& Nicolson (1963), encontrando-se 20 esporos por $40 \mathrm{~cm}^{3}$, com predominância da espécie Glomus occultum Walker. O solo recebeu calagem para el evar a saturação por bases para $50 \%$, usando cal cário dol omítico (PRNT = 95\%), foi umedecido e incubado por duas semanas, quando foi, novamente, seco e armazenado até ser usado nos experimentos, descritos a seguir: 
Exp. 1: Doses de fósforo e inoculação com G. etunicatum - Este experimento constou da adição das doses de $0,30,60,120$ e $480 \mathrm{mg} \mathrm{kg}^{-1}$ de $\mathrm{P}$ no solo na forma de superfosfato simples, combinadas com presença e ausência de inoculação com o fungo micorrízico Glomus etunicatum Becker \& Gerdemann (I e NI). As doses de P foram incorporadas ao solo, sendo este incubado, por 15 dias, em vasos com $5 \mathrm{dm}^{3}$ de solo peneirado e não fumigado, quando os teores de $\mathrm{P}$ extraídos pelo Mehlich-1 foram de 2, 9, 21, 50 e $258 \mathrm{mg} \mathrm{kg}^{-1}$ deP no solo, e os teores de cálcio extraídos pelo $\mathrm{KCl} 1 \mathrm{~mol} \mathrm{~L}-1$ de 31, 33, 38, 43 e $53 \mathrm{mmol}_{\mathrm{C}} \mathrm{dm}^{-3}$. Obteve-se material fúngico de vaso de cultivo com Brachiaria decumbens procedente da coleção de fungos MAs do DCS-UFLA. Para a inoculação, foram aplicados $5 \mathrm{~cm}^{3}$ de inóculo de solo por vaso, suficiente para fornecer 400 esporos/planta, diretamente sobre as radicelas durante a repicagem das plântulas, as quais foram obtidas a partir de sementes prégerminadas em germinador com controle de temperatura e umidade ( $25^{\circ} \mathrm{C}, 80 \% \mathrm{UR}$ ), utilizando papel de germinação. Todos os tratamentos sem inoculação receberam $10 \mathrm{~cm}^{3}$ de filtrado isento de propágulos de fungos MAs, obtidos dos vasos de cultivo dos fungos MAs.

Utilizou-se delineamento inteiramente casualizado (DIC), com quatro repetições, em esquema fatorial $5 \times 2$ (cinco doses de $\mathrm{P}$ e presença ou ausência de inoculação), sendo esses tratamentos estudados, separadamente, na trema (Trema micrantha L.) Blum.) enofedegoso (Senna macranthera Rich.). Após a repicagem das plântulas, a umidade foi ajustada para $50 \%$ do volume total de poros (VTP) e, assim, mantida durante todo o experimento por meio de pesagens periódicas. Foram feitas aplicações quinzenais de $\mathrm{N}$, na forma de sulfato de amônio e uréia, totalizando $115 \mathrm{mg} \mathrm{kg}^{-1}$ de solo e $200 \mathrm{mg} \mathrm{kg}^{-1}$ desolo, respectivamente, para a trema efedegoso. Nas doses de 0 e $30 \mathrm{mg} \mathrm{kg}^{-1}$ de $\mathrm{P}$, as duas primeiras aplicações foram de sulfato de amônio, visando a igualar a quantidade de $\mathrm{S}$ entre os tratamentos. Avaliaram-se, periodicamente, a altura e o diâmetro das plantas e, aos 75 dias, na trema, e aos 150 dias, no fedegoso, a parte aérea foi cortada e submetida à secagem em estufa a $80^{\circ} \mathrm{C}$ para obtenção da massa seca da parteaérea, sendo esse material moído eseus nutrientes determinados. As raízes foram retiradas dos vasos, separando-se $1 \mathrm{~g}$ de raízes finas para avaliação da col onização mi corrízica, eo restantefoi secoem estufa para obtenção do peso de massa seca de raízes.

Exp. 2: Adição de N, P e inoculação com G. etuni catum efumigação do solo- E sse experimento foi desenvolvido com a mesma amostra de solo do experimento 1, com fumigação $(F)$ e sem fumigação (NF). A fumigação foi feita com Bromex (brometo de metila $98 \mathrm{~g} \mathrm{~L}^{-1}$ +doropicrina $2 \mathrm{~g} \mathrm{~L}^{-1}$ ), na base de $393 \mathrm{~cm}^{3}$ de solo, objetivando-se eliminar os propágulos de fungos MAs e prevenir a micorrização pelos fungos indígenas. $\mathrm{O}$ experimento constou dos seguintes tratamentos de fertilização: Test. (testemunha); +P (adição de $60 \mathrm{mg} \mathrm{kg}^{-1}$ de $\mathrm{P}$ no solo na forma de superfosfato simples); +N (adição de $125 \mathrm{mg} \mathrm{kg}^{-1}$ de $\mathrm{N}$ nosol o aplicado em cobertura, intercalando sulfato de amônio e uréia); +PN (adição de P e N, conforme especificado nos tratamentos anteriores). Esses tratamentos foram combinados com presença e ausência de G. etunicatum (I e NI), em solo F e NF, sendo a inoculação feita como no experimento 1.

Utilizou-se um DIC, com quatro repetições, em esquema fatorial $4 \times 2 \times 2$, e a unidade experimental constituída de tubos de PVC revestidos com sacos plásticos com capacidade de $1 \mathrm{dm}^{3}$ de solo. Os tratamentos, como no experimento 1, foram testados, separadamente, na trema e no fedegoso. Foram feitas avaliações periódicas dealtura e diâmetro das plantas e, aos 79 dias, na trema, e 85 dias no fedegoso, a parte aérea e as raízes foram coletadas, e submetidas aos mesmos procedimentos relacionados no experimento 1.

Análises - Além das avaliações periódicas de diâmetro do caule, após o corte das plantas, determinaram-se a massa seca de parte aérea, massa seca de raízes eárea foliar, sendo esta última cal cul ada conforme Gomideet al. (1977). Na massa seca da parte aérea moída, determinaram-se os teores de N, P, Ca e $\mathrm{S}$. Os extratos dos tecidos foram preparados por digestão nitroperclórica (Hunter, 1975). Os teores de $\mathrm{N}$ foram determinados pelo método de destilação e titulação (Bremner \& Edwards, 1965); os de P, por colorimetria (azul de molibdênio); Ca, por espectrofotometria de absorção atômica, e S, por turbidimetria (Blanchar et al., 1965).

A colonização mi corrízica foi estimada em amostras de raízes finas clarificadas com KOH $10 \mathrm{~g} \mathrm{~L}^{-1}$, col oridas com fucsina ácida (K ormanik \& McGraw, 1982) pelo método da placa quadriculada (Giovannetti \& Mosse, 1980), sob estereomicroscópio com aumento de $40 \mathrm{x}$.

Os resultados foram submetidos a análises estatísticas, utilizando-se o programa SANEST (Zonta et al., 1984).

\section{RESULTADOS E DISCUSSÃO}

\section{Resposta a fósforo e G. etunicatum em solo não fumigado}

Os efeitos da inoculação com Ge e da adição de $P$ no diâmetro do caule da trema, aos 50 e 75 dias após a repicagem (DAR), encontram-se na figura la. Foram observados, aos 50 DAR, pequenos aumentos do diâmetro do caule pela aplicação de $\mathrm{P}$, tanto nas plantas inoculadas quanto nas sem inoculação.J á aos 75 DAR, as plantas sem $\mathrm{P}$, praticamente, não aumentaram seu diâmetro do caule em relação aos 50 DAR, porém foi observada grande resposta às doses de P. As plantas inoculadas apresentaram maior diâmetro que as não inoculadas, especialmente nas doses intermediárias de P. Para o fedegoso, não foi encontrado ajuste de regressão; todavia, foi comprovado aumento significativo $(P \leq 0,05)$ para ambos os fatores estudados (Figura 1b), bem como pequeno aumento do diâmetro do caule dessa espécie com a aplicação de P e inoculação aos 50 DAR. J á aos $150 \mathrm{DAR}$, foram observados grandes aumentos de 
diâmetro com a menor dose de $\mathrm{P}$, independentemente da inoculação, sem acréscimo nas doses mais elevadas, porém o efeito da inoculação continuou muito pequeno, refletindo a alta capacidade de crescimento do diâmetro dessa espécie neste período.

Efeitos dos tratamentos em outros parâmetros de crescimento também foram observados na trema (F igura 2). Para a produção de massa seca eárea foliar, plantas não inoculadas responderam, linearmente, à adição de P, eas inoculadas responderam mais a doses menores deste nutriente, evidenciando a eficiência da espécie fúngica em promover o crescimento da trema, por meio de seu efeito na resposta a P. A máxima produção de massa seca de parte aérea foi obtida na dose $400 \mathrm{mg} \mathrm{kg}^{-1}$ de $\mathrm{P}$ no solo. Plantas inoculadas atingiram $80 \%$ da produção máxima com apenas $100 \mathrm{mg} \mathrm{kg}^{-1}$ de $\mathrm{P}$, e plantas não inoculadas colonizadas pelos fungos indígenas produziram apenas 30\% do máximo e, para atingir $80 \%$ do máximo, requereram $320 \mathrm{mg} \mathrm{kg}^{-1}$ de $\mathrm{P}$ no solo, ou 3,2 vezes mais $\mathrm{P}$ que as plantas inoculadas. A área fol iar (Figura 2b) ea massa seca de raízes (Figura 2c) comportaram-se de maneira semel hante, confirmando, assim, os benefícios do P e da inoculação com Ge para o crescimento inicial de mudas de trema, que, como outras espécies pioneiras, apresenta elevado requerimento externo de $\mathrm{P}$ no solo (Gonçalves et al., 1992).

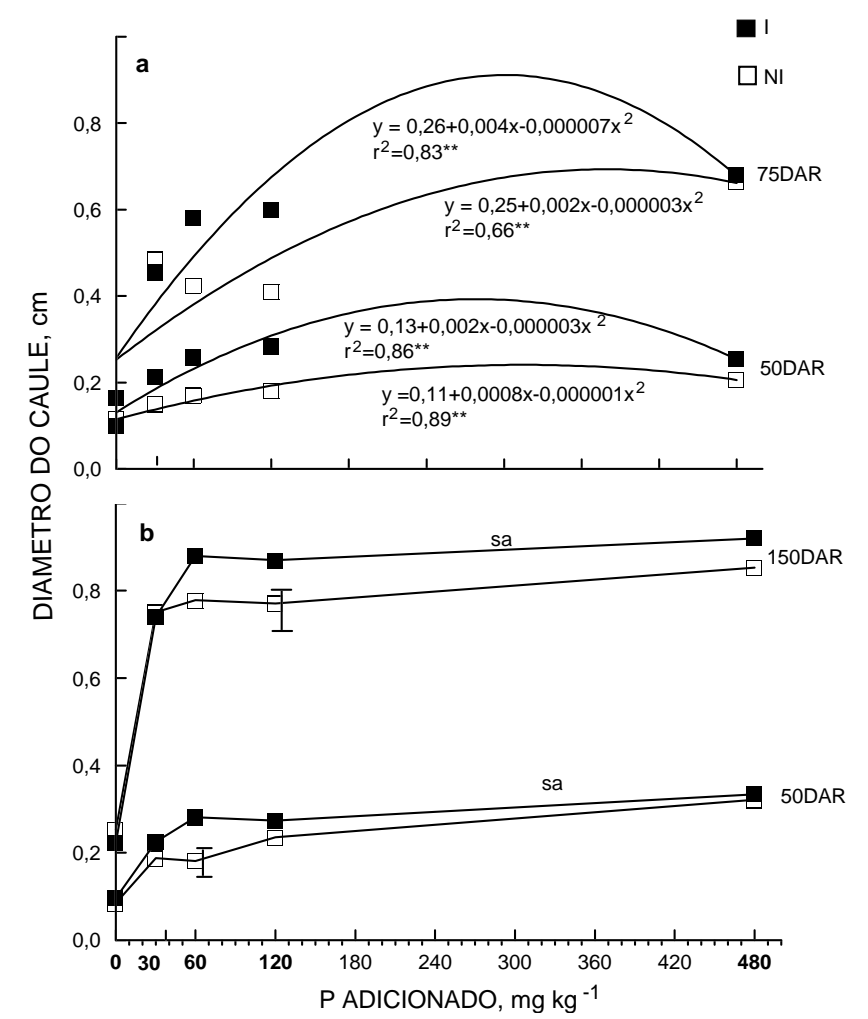

Figura 1. Diâmetro do caule da trema (a) e do fedegoso (b) em resposta a doses de superfosfato e à inoculação com o fungo micorrízico Glomus etunicatum (I- inoculado; NI - não inoculado), avaliado em diferentes dias após a repicagem (DAR). sa- sem ajuste de regressão para os modelos estudados. Barras verticais indicam a DMS a 5\% para nível de $P$.
A colonização micorrízica da trema foi elevada e influenciada pela adição de P (F igura 2d), al cançando um máximo de $75 \%$ na dose 220 , decrescendo na dose mais elevada. Nas plantas não inoculadas, a colonização pelos fungos indígenas atingiu valores próximos a $40 \%$, porém, pelo tipo de resposta verificada para crescimento, isto parece não ter contribuído para a planta hospedeira. A redução da colonização nas altas doses de $\mathrm{P}$ é comumente encontrada para outras espécies vegetais (Siqueira et al., 1984), sendo relacionada com o estado nutricional da planta hospedeira. Plantas bem supridas em nutrientes revelam mecanismos para a redução da massa ou atividade do fungo na raiz, o que seria um custo energético sem retorno para seu crescimento. De fato, em condições de alto P, plantas inoculadas produziram menos massa seca que as não inoculadas (Figura 2a). A inoculação aumentou os teores de $\mathrm{P}$ eCa (Figura 2e, f), porém, nos teores de P, isto só ocorreu para a dose de P superior a $120 \mathrm{mg} \mathrm{kg}^{-1}$ de P. Como os teores desse nutriente na parte aérea foram muito baixos, e as plantas responderam muito a pequenas doses de $\mathrm{P}$, isto pode ser decorrente de efeitos de diluição de $P$ nos tecidos das plantas inoculadas. Pelas respostas verificadas, os aumentos na absorção de Ca também podem explicar os efeitos da mi corrização no crescimento da trema. Os efeitos das doses de $\mathrm{P}$ nos teores de Ca são explicados pela
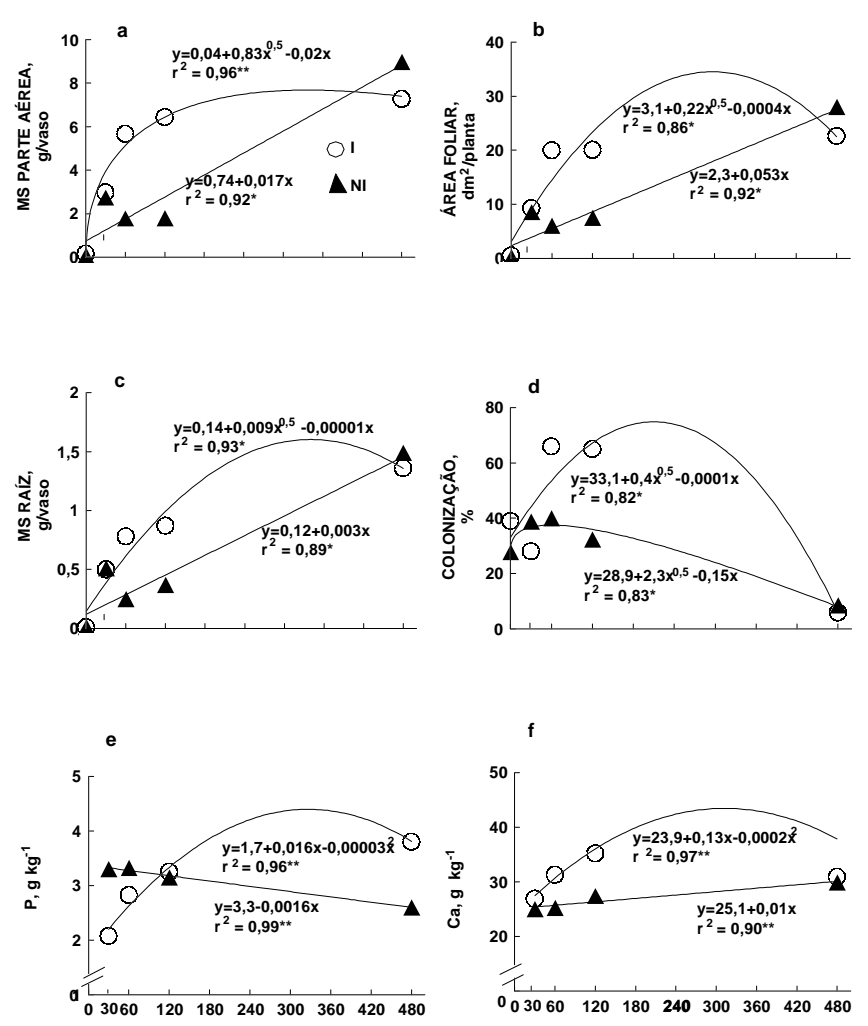

PADICIONADO, $\mathrm{mg} \mathrm{kg}^{-1}$

Figura 2. Respostas da trema a doses de superfosfato e à inoculação com o fungo mi corrízico $\mathbf{G}$. etuni catum (I - inoculado; NI-não inoculado) em latossolo vermelho-escuro de baixa fertilidade natural. 
sua presença no superfosfato simples, utilizado, como fonte de fósforo. O alto teor de Ca encontrado na massa seca de parte aérea da trema confirma sua ação de pioneira na sucessão florestal e deve também ser considerado diante do potencial forrageiro (Carvalho, 1994).

O fedegoso também apresentou elevada resposta a $\mathrm{P}$, porém baixa resposta à inoculação com Ge (F igura 3). As respostas acentuadas a $P$ nas doses mais baixas evidenciam a elevada capacidade de absorver $P$ do sol o ea baixa resposta à inoculação com Ge. A máxima produção de massa seca de parte aérea foi obtida na dose $260 \mathrm{mg} \mathrm{kg}^{-1}$ de $\mathrm{P}$ no solo. Plantas inoculadas atingiram $80 \%$ da produção máxima com $80 \mathrm{mg} \mathrm{kg}^{-1}$ de $\mathrm{P}$ no solo, enquanto as plantas não inoculadas colonizadas pelos fungos indígenas produziram $70 \%$ do máximo, requerendo $120 \mathrm{mg} \mathrm{kg}^{-1}$ de $\mathrm{P}$, ou seja 1,5 vez mais $P$ que as plantas inoculadas, para atingir 80\% do máximo. Verificaram-se respostas semel hantes para massa seca de raízes (Figura 3b).

Assim como verificado para a trema, a col onização do fedegoso foi el evada e favorecida pela adição de P
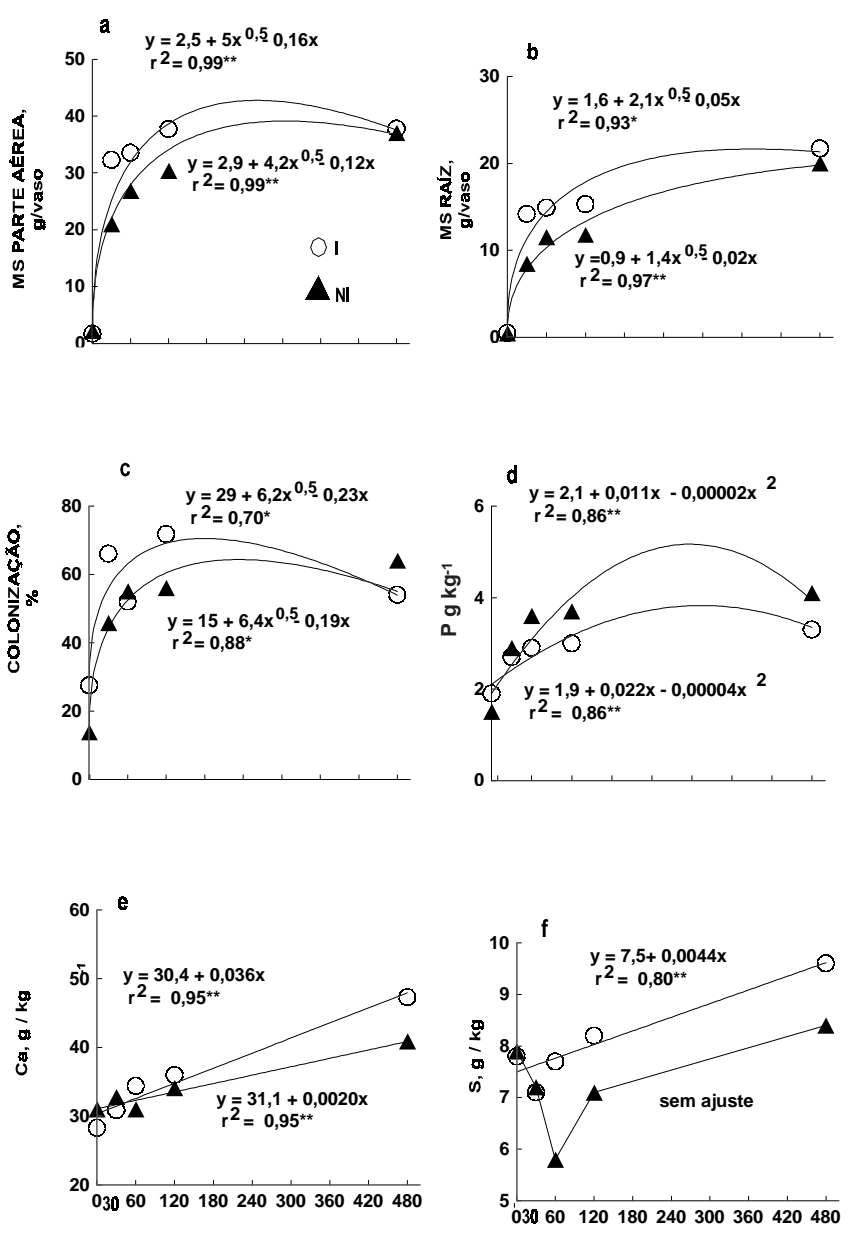

P ADICIONADO, $\mathrm{mg} \mathrm{kg}^{-1}$

Figura 3. Respostas do fedegoso a doses de superfosfato e à inoculação com o fungo micorrízico G. etunicatum (I - inoculado; NI - não inoculado) em latossolo vermelho-escuro de baixa fertilidade natural. até a dose $120 \mathrm{mg} \mathrm{kg}^{-1}$; por outro lado, foi pouco influenciada pela inoculação com Ge (Figura 3c). Mesmo com baixa densidade de propágulos originais no solo (<1 esporo $\mathrm{cm}^{-3}$ de solo), talvez, por ter essa espécie crescido por 150 dias, os fungos indígenas tenham atingi do colonização próxima daquela obtida nas plantas inoculadas, diminuindo os efeitos da inoculação. Os efeitos da inoculação nos teores de P (Figura 3d) também demonstram a ineficiência do Ge ou a eficiência superior dos fungos indígenas para essa espécie, ao contrário do que foi observado para a trema. Os teores de $\mathrm{P}$ na parte aérea aumentaram com a aplicação de superfosfato, assim como os de Ca e S (Figura 3d, e, f). Embora a aplicação de P tenha elevado os teores de $P$ na planta e aumentado seu crescimento, o fedegoso respondeu muito pouco à inoculação com Ge e, como os teores de $P$ na massa seca não foram aumentados, os aumentos nos teores de S (Figura 3f) poderiam explicar os efeitos da inoculação (Siqueira, 1994).

Diferentemente da maioria das espécies com alto grau de micotrofia (Siqueira, 1990), a elevação nas doses de $P$ teve efeito muito pequeno na colonização dofedegoso. Espécies de plantas com maior capacidade de absorção e translocação de $P$, via raízes ou micorrizas, são mais eficientes no controle da colonização micorrízica, ao contrário daquelas pouco eficientes em absorver $\mathrm{P}$ do sol o (Siqueira et al., 1984). I sto pode explicar porque o fedegoso, assim como o cafeeiro (Siqueira \& Collozzi-Filho, 1986), apresenta alta colonização mesmo em níveis elevados de $\mathrm{P}$ no substrato. Apesar de o período de crescimento experimental ter sido diferente, essas espécies apresentaram semelhante resposta a $P$, porém muito diferente à inoculação com $\mathrm{Ge}$, indicando resposta diferenciada destas ao P e à micorrização. Isso está relacionado com a demanda da espécie nesse nutriente e com a capacidade da micorriza em suprir esta demanda.

\section{Efeito de N, P, G. etunicatum e fumigação}

Verificou-se efeito para os fatores estudados, bem como para suas interações na produção de massa seca (MS) e colonização micorrízica da trema (Figura 4). A produção de MS da parte aérea e raízes foi, marcadamente, estimulada pela adição de $P$ ou PN, porém não favoreci da pela adição de apenas N (Figura $4 a, b)$. Plantas inoculadas apresentaram maior produção de MS de parte aér ea queas não inoculadas, tanto nos tratamentos $+P$ quanto nos + PN, mesmo em solo NF, onde existiam esporos de fungos MAs indígenas. Na testemunha, que não recebeu adição de $P$, a inoculação teve efeito significativo apenas na produção de MS de parte aérea do tratamento fumigado, sem a competição dos fungos indígenas. A MS de raízes também foi maior em plantas inoculadas, porém apenas nos tratamentos + PN. A fumigação aumentou a MS departeaérea das plantas inoculadas, exceto no tratamento $+\mathrm{N}$. I sto parece ser devido à eliminação dos fungos MAs indígenas que poderiam competir com o fungo introduzido e aos seus efeitos na absorção de N (Pereira et al., 1996a), embora os 
teores de $\mathrm{N}$ na MS não tenham diferido ao final do experimento. Nos tratamentos não inoculados, a fumigação reduziu o crescimento, indicando certa contribuição dos fungos indígenas para a trema.

A colonização da trema foi alta nas plantas inoculadas, exceto nos tratamentos $+\mathrm{N}$ em solo $\mathrm{F}+\mathrm{PN}$ em solo NF (Figura 4c). Por outro lado, a col onização pelos fungos indígenas (plantas não inoculadas em solo NF ) não variou entre os tratamentos deadubação, ficando próxima de $30 \%$. A aplicação de +PN reduziu a col onização das plantas no solo NF, enquanto, com aplicação de apenas $P$, não houve efeito. A col onização pel os indígenas também influenciou o crescimento da trema, porém em menor grau que o verificado para Ge. Os efeitos verificados resultam da elevação do potencial de inóculo do solo e indicam a elevada eficiência simbiótica desse fungo.

Os fatores estudados exerceram efeitos significativos nos teores foliares de $\mathrm{N}$ e $\mathrm{P}$ na trema (Figura 4d, e). Em solo fumigado, a inoculação aumentou o teor de $P$, exceto no tratamento $+N$, porém causou poucas variações no teor de $\mathrm{N}$, que foi maior apenas em plantas inoculadas que receberam esse nutriente. Quando o solo não foi fumigado, os teores de nutrientes sofreram poucos efeitos da inoculação, sendo verificada uma redução do teor de $\mathrm{N}$ nos tratamentos $+\mathrm{N}$ e +PN. Entre os tratamentos de adubação, verificou-se redução do teor de $\mathrm{P}$ na testemunha, quando em sol o fumi gado e sem inoculação, indicando aumento na absorção desse nutriente em plantas micorrizadas, em solo com baixo P. Tais diferenças refletem a complexi dade das relações entre os fatores estudados. Esses resultados indicam o alto mi cotrofismo da trema na fase inicial de crescimento; entretanto, a mi corrização não eliminou a necessidade de aplicação de fertilizante fosfatado, uma vez que plantas sem $\mathrm{P}$ cresceram pouco, mesmo quando micorrizadas. Ficou evidenciado o efeito sinérgico entre o P e a micorriza para a trema, obtido por meio da fertilização fosfatada e da inocul ação com Ge. Por outro lado, o N não foi estimulante para o crescimento dessa planta nesse sol o, que apresenta, nessas condições, suprimento adequado de $\mathrm{N}$ para essa espécie.

Os efeitos dos tratamentos sobre o crescimento e teores de $\mathrm{N}$ eP no fedegoso encontram-sena figura 5. Verificaram-se respostas semel hantes ao ocorrido para a trema, porém efeito adverso da adição de PN emaior impacto da fumigação do solo no crescimento das plantas não inoculadas (Figura $5 \mathrm{a}$, b). Os resultados indicam que o suprimento de $\mathrm{N}$ não proporcionou crescimento adicional em relação a apenas $\mathrm{P}$, apesar de essa espécie responder, negativamente, à omissão de N da adubação com NPK no campo (Lima, 1995). A baixa disponibilidade natural de $\mathrm{P}$ do solo $\left(2 \mathrm{mg} \mathrm{kg}^{-1}\right.$ deP no sol o) e o alto teor de massa orgânica (43 g kg-1) explicam esses resultados. Estudando a mesma espécie em solo com características semel hantes, Pereira et al. (1996a) não observaram efeito da adição de N, o contrário do que foi verificado em cultura com areia lavada, em que se verificaram efeitos positivos da adição de N no crescimento, o que evidencia sua demanda pelo fedegoso (Pereira et al., 1996b). A

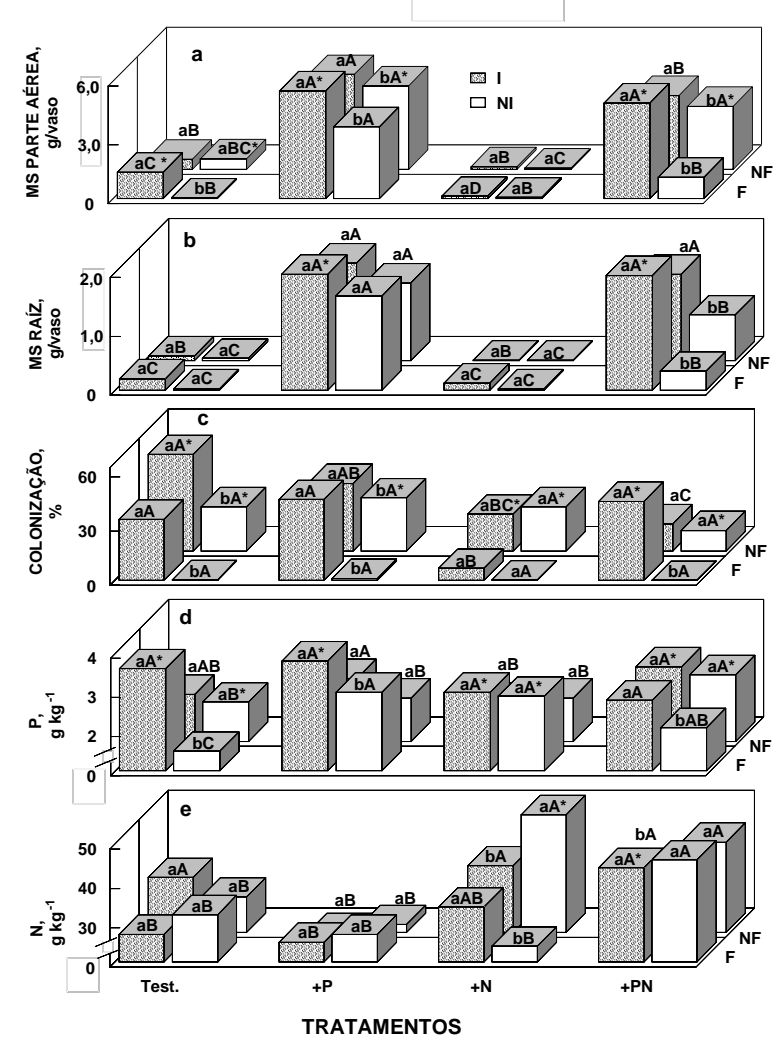

Figura 4. Parâmetros de crescimento, colonização micorrízica e teores de $\mathbf{N}$ e $\mathbf{P}$ na massa seca da parte aérea da trema em resposta à inoculação com Glomus etunicatum (I- inoculado; NI- não inoculado) em solo fumigado (F) e não fumigado (NF) e adubado com nitrogênio (N), fósforo (P) e PN. Médias seguidas pelas mesmas letras (maiúsculas comparam adubação e minúsculas comparam inoculação) não diferem pelo teste de Tukey a 5\%. E feito significativo pelo teste de Tukey a $5 \%$ para fumigação.

inoculação de $G$. etunicatum só teve efeito no crescimento, quando realizada na presença de $P$ ou PN. Apesar do efeito positivo para inoculação no tratamento $+\mathrm{P}$ no sol o NF, o crescimento das plantas não inoculadas foi próximo ao observado para plantas inoculadas. Isto confirma suspeitas levantadas no experimento 1 sobre a alta eficiência dos fungos indígenas desse sol o para o fedegoso, fato confirmado pela grande redução no crescimento das plantas não inoculadas, causada pela fumigação do solo (ver tratamento $+P$ ), que el imina a micorrização.

E mbora a trema tenha apresentado maior resposta à inoculação no primeiro experimento; no segundo, ela foi menos afetada pela eliminação dos fungos indígenas que o fedegoso, como se verifica nos tratamentos de fumi gação e inocul ação com a presença de P. Mesmo em solo com adição de P, as plantas de fedegoso não cresceram na ausência de micorrizas (solo $\mathrm{F}$, plantas não inoculadas). I sto pode ser devido a vários fatores, a saber: aspectos da relação fungos indígenas-hospedeiro, aspectos nutricionais mediados ou não pelas micorrizas (Yost \& Fox, 1979; J akobsen, 1994) e impactos biológicos e químicos da fumigação 
(Rovira, 1976; J akobsen, 1994). Aumento na disponibilidade de $\mathrm{N}$ causada pela fumigação do solo e os efeitos da micorrização na absorção deste nutriente (Pereira et al., 1996a) também podem explicar tais resultados.

Apesar de ocorrerem efeitos da interação entre os fatores na produção de massa seca, não houve este efeito para a col onização do fedegoso. A interação entre inoculação e fumigação foi significativa, porém não houve variação entre a colonização das plantas que cresceram na presença de fungos MAs, seja o Ge (inoculados) seja fungos indígenas (não inoculados). A fumigação eliminou os efeitos de fungos indígenas, resultando em ausência de colonização em plantas que cresceram em solo fumigadoe nãoinoculado. $\mathrm{Na}$ figura 5c, estão apresentados os efeitos da interação entre mi corrização e adubação das plantas. Para a trema, a inoculação aumentou a col onização, exceto nas plantas adubadas com apenas $\mathrm{N}$, que se mostrou inibitório. As plantas não inoculadas tiveram colonização próxima a $30 \%$, e os efeitos do $P$ foram semelhantes aos relatados no primeiro experimento e na trema.

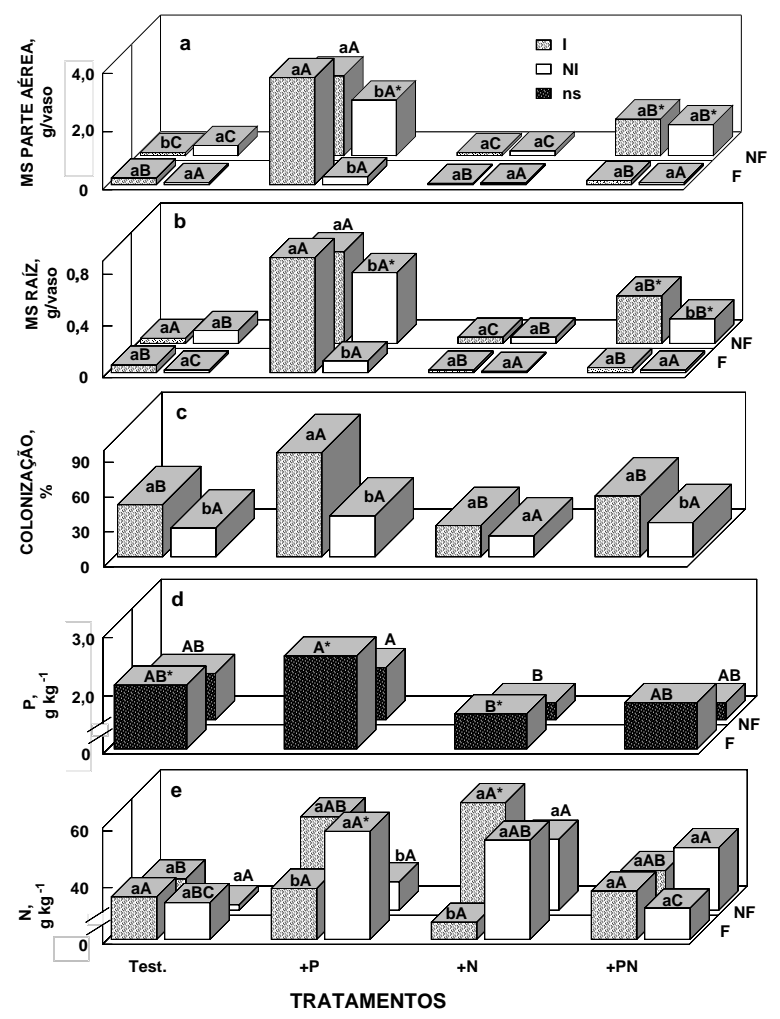

Figura 5. Parâmetros de crescimento, colonização micorrízica e teores de nutrientes na massa seca aér ea do fedegoso em resposta à inoculação com Glomus etunicatum (I- inoculado; NI-não inoculado) em solo fumigado (F) ou não fumigado (NF) e adubado com nitrogênio (N), fósforo (P) e PN. As médias seguidas pelas mesmas letras (maiúsculas comparam adubação e minúsculas comparam inoculação) não diferem pelo teste de Tukey a $5 \%$ * Efeito significativo pelo teste de Tukey a $5 \%$ para fumigação.
Os teores de $\mathrm{P}$ na massa seca aérea do fedegoso foram maiores em solo $\mathrm{F}$ e tiveram ligeiro aumento no tratamento $+P$, sem apresentar efeitos da inoculação (Figura $5 d$ ). I sto resulta da complexa interação entre os fatores estudados. Apesar da alta resposta à inoculação, neste tratamento, em termos de MS, isto não foi detectado na análise de variância para teor de P. Os teores de N variaram bastante, tendo apresentado as plantas não inoculadas grande aumento desse nutriente nos tratamentos $+\mathrm{P}$ e $+\mathrm{N}$, em solo $\mathrm{F}$; já, em solo não fumigado, a inoculação afetou o teor de $\mathrm{N}$ apenas no tratamento + P, com redução para plantas inoculadas (Figura $5 d$ ). Essas variações ocorreram pela inoculação e adubação das plantas, mas a fumi gação do sol o também contribuiu, aumentando a disponibilidade de nutrientes no solo ou eliminando os fungos indígenas.

Comparando o comportamento das duas espécies, verifica-se que, enquanto a trema é altamente responsiva ao fungo $G$. etunicatum, mesmo na presença de fungos indígenas, o fedegoso mostra pequena resposta a esse fungo, alcançando crescimento satisfatório em solo apenas com os fungos indígenas. Se, por um lado, a inoculação teve efeito diferente entre as espécies estudadas, a resposta à apl icação de P evidencia a grande demanda de mudas dessas duas espécies e a necessidade de adubação em solo com baixa disponibilidade desse nutriente. $\mathrm{A}$ adição de N, por sua vez, não teve efeito benéfico no crescimento, possivelmente pel ofato de o sol ojá conter quantidades adequadas. A redução no crescimento apresentada pelo fedegoso em solo fumigado é atribuída à eliminação dos fungos indígenas no tratamento $+\mathrm{P}$ eao aumento na disponibilidade de $\mathrm{N}$ que, no tratamento $+\mathrm{PN}$, reduziu a colonização micorrízica.

\section{CONCLUSÕES}

1. A inoculação com G. etunicatum em solo não fumigado exerce efeito diferenciado sobre as espécies, com alta resposta na trema em doses intermediárias deP e pequeno efeito no fedegoso, para o qual os fungos indígenas são eficientes.

2. Plantas de trema e fedegoso col onizadas por $\mathrm{G}$. etuni catum atingem $80 \%$ do crescimento máximo em solo não fumigado com 100 e $80 \mathrm{mg} \mathrm{kg}^{-1}$ de $\mathrm{P}$ no solo, respectivamente, enquanto as plantas sem o fungo requerem, respectivamente, 3,2 e 1,5 vezes mais fósforo para atingir tal crescimento.

3. Sem a aplicação de fósforo, mesmo inoculadas com G. etunicatum, as espécies têm crescimento reduzido, indicando el evada deficiência desse nutriente no solo estudado. Por outro lado, nem a trema nem o fedegoso respondem à adi ção de apenas $\mathrm{N}$ mineral neste solo.

4. Adição de fósforo e inoculação com o fungo micorrízico G. etunicatum facilitam o crescimento da trema e do fedegoso em solo de baixa fertilidade natural. 


\section{LITERATURA CITADA}

BLANCHAR, R.W.; REHM, G. \& CALDWELL,A.C. Sulfur in plant material digestion with nitric and percloric acid. Soil Sci. Soc. Am. Proc., Madison, 29:71-72, 1965.

BREMNER, J.M. \& EDWARDS, H.P. Determination and isotope ratio analyses of different forms of nitrogen in soils. I. Aparatus and procedures for destilation and determination for amonium. Soil Sci. Soc. Am. Proc., Madison, 29:504-507, 1965.

CARNEIRO, M.A.C.; SIQUEIRA, J .O.; DAVIDE, A.C.; GOMES, L.J .; CURI, N. \& VALE, F.R. Colonização micorrízica, crescimento e teores de nutrientes em trinta e uma espécies arbóreas em resposta a fungo micorrízico e superfosfato simples. Scientia Forestalis, Piracicaba, 50:21-36, 1996.

CARVALHO, P.E.R. Espécies florestais brasileiras: recomendações silviculturais, potencialidades e uso da madeira. Brasília, EMBRAPA-CNPF, 1994, 580p.

COLONNA, J .P.; THOEN, D.; DUCOUSSO, M. \& BRADJI, S. Comparative effects of Glomus mosseae and $P$ fertilizer on foliar mineral composition of Acacia senegal seedlings inoculated with Rhyzobium. Mycorrhiza, Berlin, 1:35-38, 1991.

FRANCO, A.A. Fixação de nitrogênio em árvores e fertilidade do solo. Pesq. agropec. bras., Brasília, 19:253-261, 1984.

GERDEMANN, J.W. \& NICOLSON, T.H. Spores of mycorrhizal Endogone species extracted from soil by wet sieving and decanting. Trans. Brit. Mycol. Soc., London, 46:235-244, 1963.

GIOVANNETTI, M. \& MOSSE, B. An evaluation of techniques to measure vesicular-arbuscular mycorrhizal infection in roots. New Phytol., London, 84:484-500, 1980.

GOMIDE, M.B.; LEMOS, O.V.; TOURINO, D.; CARVALHO, M.M.; CARVALHO, J.G. \& DUARTE, C.S. Comparação entre métodos de determinação de área foliar em cafeeiros 'M undo Novo e Catuaí'. Ci. Prát., Lavras, 1:118-123, 1977.

GONÇALVES, J.L. de M.; KAGEYAMA, P.Y.; FREIXÊDAS; GONÇALVES, J.C. \& GERES, W.L. de A. Capacidade de absorção e eficiência nutricional de al gumas espécies arbóreas tropicais. In: CONGRESSO NACIONAL SOBRE ESSÊ N CIAS NATIVAS, 2., São Paulo, 1992. Anais. São Paulo, Instituto Florestal, 1992. p.463-469.

HUNTER, A.H. Laboratory analisys of vegetal tissues samples. Raleigh, Internacional Soil Fertility Evaluation and Improvement Program, N.C.S.U., 1975. 5p.

J AKOBSEN, I. Research approaches to study the functioning of vesicular-arbuscular mycorrhizas in the field. Plant Soil, The Hague, 159:141-147, 1994.

KORMANIK, P.P. \& MCGRAW, A.C. Quantification of vesiculararbuscular mycorrhizal in plants roots. In: SCHENCK, N.C., ed. Methods and principles of mycorrhizal research, St. Paul, American Phytopathological Society, 1982. p.37-46.
LIMA, H.N. Crescimento inicial de sete espécies arbóreas nativas em resposta à adubação com NPK a campo. Lavras, ESAL, 1995. 53p. (Dissertação de Mestrado)

LORENZI, H. Árvores brasileiras: manual de identificação ecultivo de plantas arbóreas nativas do Brasil. Nova Odessa, Plantarum, 1992. 352p.

PEREIRA, E.G.; SIQUEIRA, J .O.; CURI, N.; MOREIRA, F.M.S. \& PURCINO, A.A.C. Efeitos da micorriza e do suprimento de fósforo na atividade enzimática e na resposta de espécies arbóreas ao nitrogênio. R. bras. Fisiol. Veg., Campinas, 8:5965, 1996a.

PEREIRA, E.G.; SIQUEIRA, J .O.; VALE, F.R. \& MOREIRA, F.M.S. I nfluência do nitrogênio mineral no crescimento e col onização micorrízica de mudas de árvores. Pesq. agrop. bras., Brasília, 31:653-662, 1996b.

RENÓ, N.B.; SIQUEIRA, J .O.; CURI, N. \& VALE, F.R. Limitações nutricionais ao crescimento inicial de quatro espécies arbóreas nativas em latossol o vermel ho-amarelo. Pesq. agrop. bras., Brasília, 32:17-25, 1997.

ROVIRA, A.D. Studies on soil fumigation- I Effects on ammonium, nitrate and phosphate in soil and on the growth, nutrition and yield of weat. Soil Biol. Biochem., Oxford, 8:241-247, 1976.

SIQUEIRA, J.O. Eficiência de fertilizantes fosfatados em associações micorrízicas. In: ENCONTRO NACIONAL DE ROCHA FOSFÁTICA, 5., Anais. São Paulo, I BRAFOS, 1990. p.165-193.

SIQUEIRA, J.O. Micorrizas arbusculares. In: ARAUJ O, R.S. \& HUNGRIA, M., eds. Microrganismos de importância agrícola. Brasília, EMBRAPA, 1994. p.151-194.

SIQUEIRA, J.O. \& COLLOZZI-FILHO, A. Micorrizas vesículoarbusculares em mudas de cafeeiro. II. Efeito do fósforo no estabelecimento efuncionamento. R. bras. Ci. Solo, Campinas, 10:207-211, 1986.

SIQUEIRA, J.O.; HUBBELL, D.H. \& VALLE, R.R. Effects of phosphorus on formation of the vesicular-arbuscular mycorrhizal symbiosis. Pesq. agropec. bras., Brasília, 19:1465-1474, 1984.

SIQUEIRA, J.O.; CURI, N.; VALE, F.R.; FERREIRA, M.M. \& MOREIRA, F.M.S. Aspectos de solos, nutrição vegetal e microbiologia na implantação de matas ciliares. Belo Horizonte, CEMIG/UFLA, 1995, 28p.

ST. J OHN, T.V. Uma lista de espécies de plantas tropicais brasileiras naturalmente infectadas com micorrizas vesicular-arbuscular. Acta Amaz., Manaus, 10:229-234, 1980.

YOST, R.S. \& FOX, R.L. Contribuition of mycorrhizaeto P nutrition of crops growing on an Oxisol. Agron. J., Madison, 71:903908, 1979.

ZONTA, E.P.; MACHADO, A.A. \& SILVEIRA-J ÚNIOR, P. Sistema de Análise Estatística para Microcomputadores (SANEST). Pelotas, 1984, 151p. 